\title{
An iatrogenic single-loop macroreentrant biatrial tachycardia developing after persistent atrial fibrillation ablation
}

\author{
Jian Zhou ${ }^{1}$, Jinbo $\mathrm{Yu}^{1}$, Xiaorong $\mathrm{LI}^{1}$, and Bing YANG ${ }^{1}$ \\ ${ }^{1}$ Shanghai East Hospital
}

June 10, 2020

\begin{abstract}
Biatrial macro-reentrant tachycardia (Bi-MAT) is a relatively uncommon arrhythmia. Sometimes, it can be produced by the left atrial (LA) anterior or septal-anterior linear ablation. However, data concerning the proarrhythmic role of this ablation lesions are lacking. Here, we reported a case of a single-loop Bi-MAT developing after the LA anterior ablation line performed in one patient with persistent atrial fibrillation.
\end{abstract}

\section{Introduction}

Extensive left atrial ablation beyond pulmonary vein isolation (PVI) in the treatment of persistent AF is associated with a high incidence of iatrogenic atrial tachycardia (AT). The most possible mechanisms of these tachycardias are macro- or micro-reentries, and the reentry circuits mostly confined to the left atrium. Bi-MAT is a relatively-rare reentrant type which reported previously. But notice, a recent study found that Bi-MATs developed in $31 \%$ of persistent AF cases when the left atrial anterior linear ablation is performed. ${ }^{1}$ To date, there are only sporadic reports of Bi-MAT during or post AF ablation. Herein, we present a case of a single-loop Bi-MAT, which developed in persistent AF catheter ablation.

\section{Case report}

A 70-year-old male was referred for catheter ablation of persistent drug-resistant AF. He had a 5-year history of symptomatic AF, which had been persistent for the last 6 months. The initial AF ablation procedure consisted of PVI, linear ablation of the cavotricuspid isthmus (CTI), then complex fractionated electrogrambased ablation at the anterior and posterior LA and a linear ablation at the LA roof were completed, but $\mathrm{AF}$ was not terminated and external electrical cardioversion (using one 150J biphasic shock) was required to restore sinus rhythm. Unfortunately, he had a recurrence after a one-year follow-up, the electrocardiogram (ECG) during clinical symptoms revealed a regularity AT (atrial cycle length 270ms) with 2:1 atrioventricular conduction. The $\mathrm{P}$ waves in the inferior and precordial leads $\mathrm{V}_{1}-\mathrm{V}_{3}$ were positive, suggesting LA originated; Leads I, aVL, and $\mathrm{V}_{4}-\mathrm{V}_{6}$ showed either a flat or low-amplitude upright component; and there was an isoelectric segment between $\mathrm{P}$ waves (Figure $1 \mathrm{~A}$ ).

In the redo procedure, a quadripolar catheter was placed into the coronary sinus (CS) and it demonstrated a consistent proximal-to-distal activation pattern during the tachycardia. Counterclockwise peri-mitral atrial flutter (PMAFL) was confirmed by classical entrainment criteria. Then 3-dimensional detailed LA endocardial electroanatomic mapping was performed during the PMAFL, extensive low-voltage zones (LVZs) with fractionated electrograms, caused by spontaneous scarring and prior ablation, were observed on the anterior and posterior wall. The reinforced defragmentation ablation was performed in the initial posterior and anterior ablation area, and a linear ablation at the LA anterior wall (connecting right PV roof to the anterior mitral annulus) were completed, which prolonged the AT cycle length to $305 \mathrm{~ms}$ (Figure $1 B$ ). Next, entrainment pacing and activation mapping were performed again to identify the reentry circuit, and it 
turned out that the changed AT with a biatrial macro-reentrant circuit using most of the mitral annulus in the LA circuit (including the anterolateral and posterior wall but not the LA anterior septum), and the right atrium (RA) septum in the RA circuit (Figure $2 A$ and $2 B$ ). Finally, irrigated radiofrequency targeted at the endocardial breakthrough site of RA septum with a temperature setting of $43^{\circ} \mathrm{C}$, a maximum power output of $30 \mathrm{~W}$, and an infusion rate of $17 \mathrm{~mL} / \mathrm{min}$ was successfully led to termination and non-inducibility of tachycardia (Figure 3 ). Then the LA anterior linear ablation, prior PVI, LA roof, and CTI ablation lines were checked to bidirectional block.

An anterior or septal-anterior ablation line, connecting right pulmonary veins to the mitral annulus, has been suggested as an alternative to mitral isthmus (MI) ablation for PMAFL. But it can produce an obstacle to the conduction in the LA septum and might increase the risk of developing biatrial macro-reentries. ${ }^{2}$ If an electro-anatomic activation map could not identify a complete reentrant circuit in a single chamber, detailed activation mapping and entrainment pacing in both atria are required to clarify the critical reentrant circuit. Besides MI or CTI linear ablation, the Bi-MAT also can be terminated by the ablation of the breakthrough site of RA or LA septum. But notice that sequential endocardial ablation, or sometimes, bipolar ablation configuration from both sides are needed to achieve complete conduction block. ${ }^{3}$
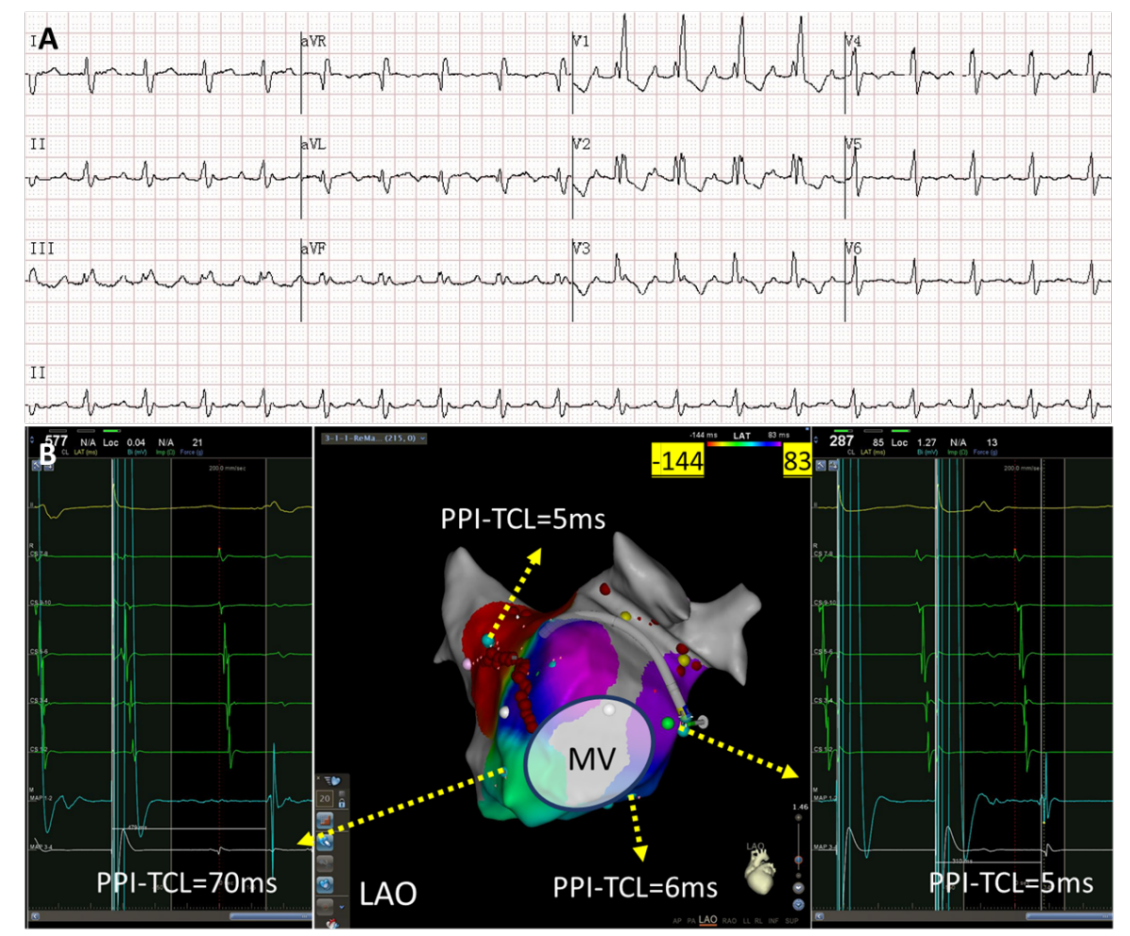

Figure 1: (A) : Surface 12-lead ECG recorded during AT with an atrial tachycardia cycle length (TCL) of 270ms; and(B) : electroanatomic mapping of a changed AT (TCL: 305ms) after LA anterior wall linear ablation, a redo activation map and entrainment could not identify a complete AT circuit around the mitral annulus, the "early" to "late" activation time in LA was $227 \mathrm{~ms}$, and a long PPI was observed in the left anterior septum (PPI-TCL: 70ms). LAO, left anterior oblique view; MV, mitral valve; PPI, post-pacing interval. 


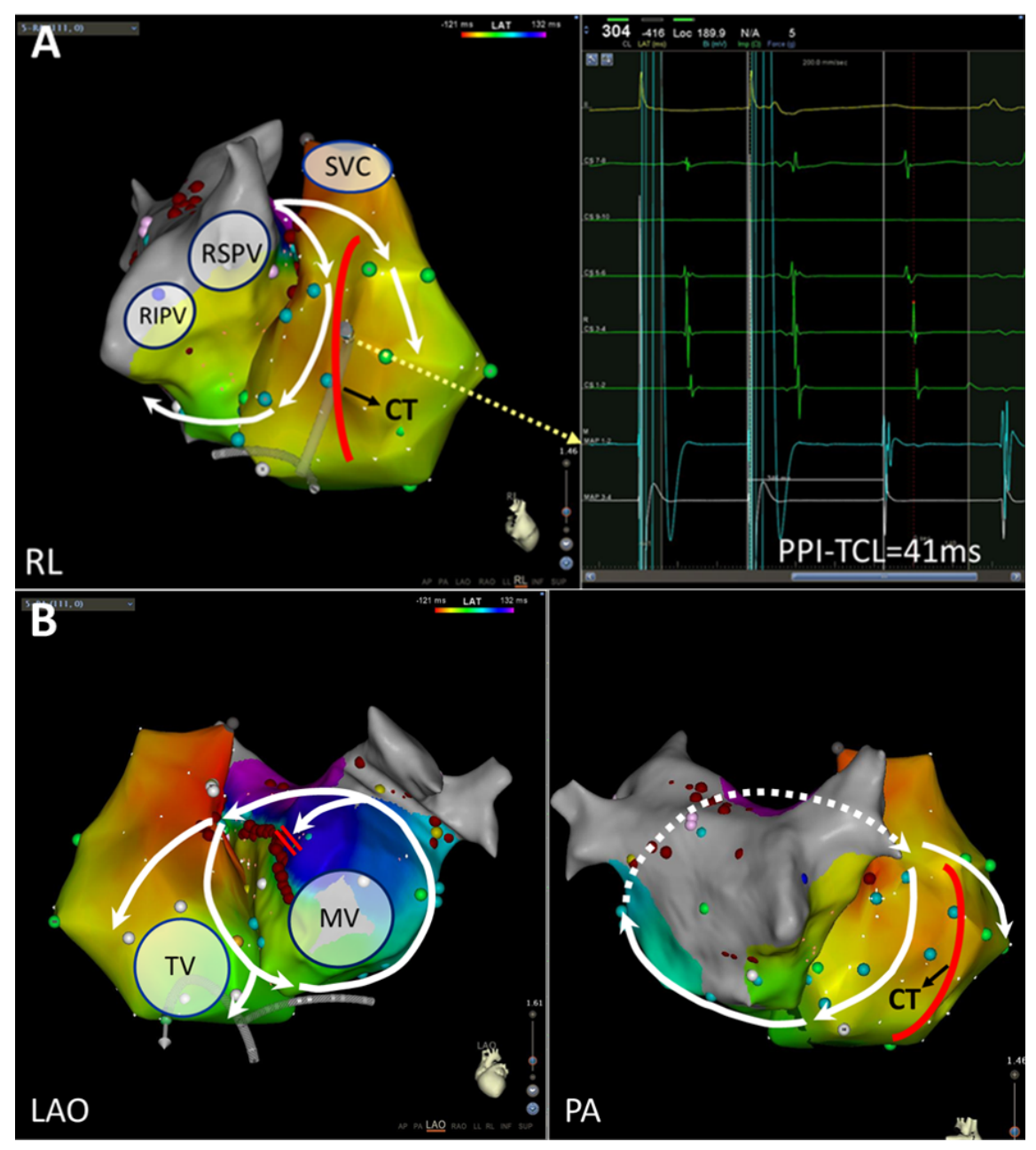

Figure 2: A and B : Detailed electroanatomic activation and entrainment mapping of the changed AT after LA anterior linear ablation, a biatrial macro-reentrant circuit using most of the mitral annulus in the LA circuit (including the anterolateral and posterior wall but not the LA anterior septum), and the RA septum in the RA circuit (excluding the RA free-wall). It showed a long post pacing interval (PPI) in front of the crista terminalis (solid red line ) (PPI-TCL: 41ms). CT: crista terminalis; PA, posteroanterior view; RL, right lateral view; RIPV, right inferior pulmonary vein; RSPV, right superior pulmonary vein; SVC, superior vena cava; TV, tricuspid valve.

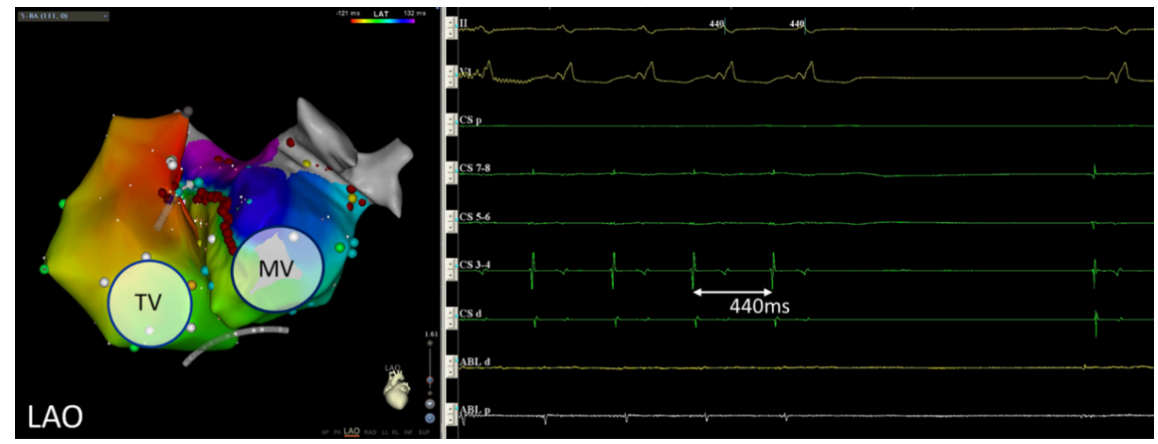


Figure 3: Electroanatomic mapping and Intracardiac recordings during Bi-MAT ablation (paper speed $100 \mathrm{~mm} / \mathrm{s}$ ); Note that the ablation catheter targeted at the endocardial breakthrough site of RA septum, radiofrequency prolonged TCL to 440ms and then successfully terminated the tachycardia.

\section{Conflict of interest}

The authors report no financial relationships or conflicts of interest regarding the content herein.

\section{References}

1. Mikhaylov EN, Mitrofanova LB, Vander MA, Tatarskiy RB, Kamenev AV, Abramov ML, et al. Biatrial tachycardia following linear anterior wall ablation for the perimitral reentry: incidence and electrophysiological evaluations. J Cardiovasc Electrophysiol . 2015;26:28-35.

2. Kitamura T, Martin R, Denis A, Takigawa M, Duparc A, Rollin A, et al. Characteristics of SingleLoop Macroreentrant Biatrial Tachycardia Diagnosed by Ultrahigh-Resolution Mapping System. Circ Arrhythm Electrophysiol . 2018;11:e005558.

3. Yu J, Chen K, Yang B, Zhang F, Ju W, Chen H, et al. Peri-mitral atrial flutter: personalized ablation strategy based on arrhythmogenic substrate. Europace. 2018;20:835-842. 